\title{
A Photon Transport Problem with a Time-Dependent Point Source
}

\author{
A Belleni-Morante \\ Dipartimento di Ingegneria Civile \\ Universita' di Firenze \\ Via S. Marta 3 \\ 50139 Firenze, Italia \\ e-mailabelleni@dicea.unifi.it \\ W Lamb and A C McBride \\ Department of Mathematics \\ University of Strathclyde \\ Livingstone Tower, 26 Richmond Street, \\ Glasgow, G1 1XH, U.K. \\ e-mails:wl@maths.strath.ac.uk; acmcb@maths.strath.ac.uk
}

May 2008

\begin{abstract}
We consider a time-dependent problem of photon transport in an interstellar cloud with a point photon source modelled by a Dirac $\delta$ functional. The existence of a unique distributional solution to this problem is established by using the theory of continuous semigroups of operators on locally convex spaces coupled with a constructive approach for producing spaces of generalized functions.
\end{abstract}

Key words: Photon transport problems, distributional solutions.

AMS subject classification: 85A25, 35R30, 46F05

Abbreviated Title: Photon Transport Problem 


\section{Introduction}

In a recent paper, [2], a stationary photon transport problem was considered in an interstellar cloud containing a time-independent UV-photon source of the form $q_{0} \delta\left(\mathbf{x}-\mathbf{x}_{0}\right)$, where $q_{0}$ is a positive constant, $\mathbf{x}_{0}$ is a location within the cloud and $\delta$ is the Dirac functional. The use of the $\delta$ functional is justified by the the fact that the diameter of the source (a star) is much smaller than the diameter of the cloud. In [2], we proved that such a transport problem has a unique distributional solution.

We recall from [3] and [4] that interstellar clouds are large astronomical objects (the diameter of an average cloud ranging from $10^{3}$ to $10^{5}$ times the diameter of our solar system) and are composed of a low density mixture of gases (mainly hydrogen) and dust grains. Interstellar clouds may contain stars which emit UV-photons in a time-independent fashion or in a time-varying manner (variable stars, with periods ranging from a few days to one year).

In this paper, our aim is to extend the work presented in [2] by considering a time-dependent photon transport problem in an interstellar cloud containing a variable star (i.e. a time-dependent point source). We begin in Section 2 by describing a mathematical model of the problem and discuss how the Banach space theory of semigroups of operators can be used to analyse the case when the time-dependent source is a "classical" function. This requires the problem to be expressed in abstract form, on an appropriate Banach space $X$, as

$$
\frac{d}{d t} N(t)=T[N(t)]+q(t), t>0 ; N(0)=N_{0},
$$

where $T$ is the infinitesimal generator of a semigroup on $X, q:[0, \infty) \rightarrow X$ is the source term, and a solution $N:[0, \infty) \rightarrow X$ is sought.

Unfortunately, the results presented in Section 2 cannot cater for time-dependent point sources represented in terms of the Dirac delta functional. However, by using a modified version of the distributional approach developed in [2] for the associated stationary problem, together with the theory of semigroups of operators on locally convex topological vector spaces, we are able to formulate a more general version of the abstract problem as

$$
\frac{d}{d t} N(t)=\tilde{T}[N(t)]+q(t), t>0 ; N(0)=N_{0} .
$$

In (1.2), $\tilde{T}$, an extension of $T$, is defined on a space of generalized functions, $Z^{\prime}$, that is constructed in a systematic manner and $q:[0, \infty) \rightarrow Z^{\prime}$ can now incorporate distributional terms such as $\delta\left(\mathbf{x}-\mathbf{x}_{0}\right)$.

A key ingredient in our approach is the existence of a suitably defined adjoint operator, $A$, of $T$ such that $A$ is the infinitesimal generator of a strongly continuous semigroup on a Banach space $Y$, consisting of continuous functions, that can be identified with a closed subspace of $X^{*}$. Relevant results on adjoint semigroups and adjoints of generators are presented in Section 3 and used to show that such an operator $A$ can always be obtained. Two separate cases are discussed. The first, which is more straightforward, is when the scattering kernel (defined in Section 2) is identically zero on the boundary of the cloud. The second, which includes the case of a constant scattering kernel, deals with kernels which do not vanish on the boundary and requires a 
perturbation result [15, Lemma 2.2] due to Suhadolc and Vidav.

As the theory of semigroups of operators in locally convex topological vector spaces is perhaps not as well-known as the Banach space version, some details are included in Section 4. In particular, we concentrate on locally convex spaces that are Fréchet spaces in which the topology is defined in terms of a countable collection of norms. Complete countably normed spaces of this type are discussed in [7] and are often used in developing the theory of generalized functions. It should also be noted that semigroups of operators defined in locally convex spaces are playing an increasingly significant role in some recent theoretical developments. One particular case is the theory of bi-continuous semigroups $[6,9]$ in which the semigroups of interest are only locally bounded on some Banach space $X$, but are strongly continuous with respect to some other locally convex Hausdorff topology on $X$.

In Section 5, we define spaces, $Z$ and $Z^{\prime}$, of test functions and generalized functions respectively, and show that $Z$ is a complete countably normed space. We also establish that a suitable extension, $\tilde{T}$, of $T$ can be defined on $Z^{\prime}$. The results of Section 3 are then applied in Section 6 to prove the existence and uniqueness of a solution $N:[0, \infty) \rightarrow Z^{\prime}$ to equation (1.2) when $q(t)=q_{0}(t) \delta\left(\mathbf{x}-\mathbf{x}_{0}\right)$. We conclude in Section 7 by indicating possible extensions of the work presented here.

It should be noted that our approach shares certain features of the method favoured by Suhadolc and Vidav [15] in which Banach spaces of regular Borel measures are used. Mention should also be made of the recent work of Lant and Thieme [11] which includes a method for obtaining a strongly continuous semigroup on a subspace, $X^{\oslash}$, of the dual $X^{*}$ of a general Banach space $X$; see [11, Proposition 6.4]. In this case, an operator $A$ on $X^{\varnothing}$ is produced that is adjoint to $T$, with $T$ only required to be the generator of a (once) integrated semigroup on $X$. However, we believe that there are some advantages to be gained by moving from a Banach space setting to that of a Fréchet space. Not least is the fact that our constructive approach guarantees that the extended operator $\tilde{T}$ is the infinitesimal generator of an equicontinuous semigroup on $Z^{\prime}$ and is also defined on all of $Z^{\prime}$.

\section{The Mathematical Model}

In what follows, we shall assume that the interstellar cloud under consideration occupies the convex region $V \subset \mathbb{R}^{3}$, bounded by the closed "regular" surface $\Sigma$, and that $V_{i}$ is the interior of $V$ so that $V=V_{i} \cup \Sigma$. Further, let $N(\mathbf{x}, \mathbf{u}, t)$ be the photon distribution function at time $t \geq 0$ and at $(\mathbf{x}, \mathbf{u})$ with $\mathbf{x} \in V$ and $\mathbf{u} \in S$, where $S$ is the surface of the unit sphere. Hence, $N(\mathbf{x}, \mathbf{u}, t) d \mathbf{x} d \mathbf{u}$ is the expected number of photons which, at time $t$, are in the unit volume element $d \mathbf{x}$ and have velocity within the solid angle $d \mathbf{u}$ around the unit vector $\mathbf{u} \in S$.

The photon distribution function $N(\mathbf{x}, \mathbf{u}, t)$ satisfies the system, [13, 14], 


$$
\begin{aligned}
& \frac{\partial N}{\partial t}(\mathbf{x}, \mathbf{u}, t)=-c \mathbf{u} \cdot \nabla_{\mathbf{x}} N(\mathbf{x}, \mathbf{u}, t)-c \sigma(\mathbf{x}) N(\mathbf{x}, \mathbf{u}, t) \\
& \quad+c \sigma_{s}(\mathbf{x}) \int_{S} k\left(\mathbf{u}, \mathbf{u}^{\prime}\right) N\left(\mathbf{x}, \mathbf{u}^{\prime}, t\right) d \mathbf{u}^{\prime}+q(\mathbf{x}, \mathbf{u}, t), \quad t>0,(\mathbf{x}, \mathbf{u}) \in V_{i} \times S, \\
& N(\mathbf{y}, \mathbf{u}, t)=0 \text { if } \mathbf{y} \in \Sigma \text { and } \mathbf{u} \cdot \mathbf{n}(\mathbf{y})<0, t>0, \\
& N(\mathbf{x}, \mathbf{u}, 0)=N_{0}(\mathbf{x}, \mathbf{u}),(\mathbf{x}, \mathbf{u}) \in V \times S .
\end{aligned}
$$

In (2.1), $c$ is the speed of light, $\sigma_{s}$ and $\sigma$ are the scattering and the total cross-sections (which take care of the interactions between photons and cloud particles), $q$ is a source term (due to the presence of one or more photon-emitting stars within the cloud), and $k$ is a scattering kernel (with $k=1 /(4 \pi)$ if scattering is isotropic). Further, (2.2) is a non re-entry boundary condition, where $\mathbf{n}(\mathbf{y})$ is the outward directed normal to $\Sigma$ at $\mathbf{y}$. Note that (2.2) holds provided that $V$ is convex, there are no photon sources outside $V$ and $\sigma_{s}(\mathbf{x})=0$ if $\mathbf{x} \notin V$.

Finally, in (2.3), $N_{0}$ is a given non-negative initial photon distribution function.

In the sequel, we shall assume that

$$
\begin{aligned}
& 0 \leq \sigma_{s}(\mathbf{x})<\sigma(\mathbf{x}) \forall \mathbf{x} \in V, \sigma_{s} \in C(V), \sigma \in C(V), \text { with } \sigma_{s, \max }<\sigma_{\min }, \\
& \text { ( where } \left.\sigma_{\min }=\min \{\sigma(\mathbf{x}): \mathbf{x} \in V\} \text { and } \sigma_{s, \max }=\max \left\{\sigma_{s}(\mathbf{x}): \mathbf{x} \in V\right\}\right), \\
& 0<k\left(\mathbf{u}, \mathbf{u}^{\prime}\right)=k\left(\mathbf{u}^{\prime}, \mathbf{u}\right), k \in L^{\infty}(S \times S), \int_{S} k\left(\mathbf{u}, \mathbf{u}^{\prime}\right) d \mathbf{u}=1 \forall \mathbf{u}^{\prime} \in S .
\end{aligned}
$$

In order to write the abstract form of the system (2.1) - (2.3) we consider the real Banach space $X=L^{1}(V \times S)$ equipped with the usual norm

$$
\|\psi\|_{1}=\int_{S} \int_{V}|\psi(\mathbf{x}, \mathbf{u})| d \mathbf{x} d \mathbf{u},
$$

and define the following operators

$$
\begin{aligned}
& (K \psi)(\mathbf{x}, \mathbf{u})=c \sigma_{s}(\mathbf{x}) \int_{S} k\left(\mathbf{u}, \mathbf{u}^{\prime}\right) \psi\left(\mathbf{x}, \mathbf{u}^{\prime}\right) d \mathbf{u}^{\prime}, \\
& \left(B_{0} \psi\right)(\mathbf{x}, \mathbf{u})=-c \mathbf{u} \cdot \nabla_{\mathbf{x}} \psi(\mathbf{x}, \mathbf{u}), \\
& (B \psi)(\mathbf{x}, \mathbf{u})=-c \mathbf{u} \cdot \nabla_{\mathbf{x}} \psi(\mathbf{x}, \mathbf{u})-c \sigma(\mathbf{x}) \psi(\mathbf{x}, \mathbf{u}), \\
& T \psi=B \psi+K \psi
\end{aligned}
$$

with respective domains

$$
\begin{aligned}
& D(K)=X \\
& D\left(B_{0}\right)=\left\{\psi \in X:-c \mathbf{u} \cdot \nabla_{\mathbf{x}} \psi \in X \text { and } \psi(\mathbf{y}, \mathbf{u})=0 \text { if } \mathbf{y} \in \Sigma \text { and } \mathbf{u} \cdot \mathbf{n}(\mathbf{y})<0\right\} \\
& D(T)=D(B)=D\left(B_{0}\right) .
\end{aligned}
$$

Note that, in $D\left(B_{0}\right)$, the differential expression $-c \mathbf{u} \cdot \nabla_{\mathbf{x}}$ should be interpreted as a directional derivative that exists almost everywhere with respect to a particular measure and the boundary condition should be expressed in terms of an appropriate trace operator; see [17, Chapters 2 and 3] for details.

Some standard results concerning the operators defined by $(2.7)-(2.13)$ are listed below $($ see $[8,17])$. 
(a) $K \in B(X)$, i.e. $K$ is a bounded operator on $X$, with

$$
\|K\|_{B(X)} \leq c \sigma_{s, \max }
$$

This follows from assumptions (2.4) and (2.6).

(b) $B_{0} \in \mathcal{G}(1,0 ; X)$, i.e. the free streaming operator $B_{0}$ is the infinitesimal generator of the contraction $C_{0}$-semigroup $\left\{\exp \left(t B_{0}\right)\right\}_{t \geq 0} ;$ see [1]. This follows from the fact that the domain $D\left(B_{0}\right)$ is dense in $X$ because $D\left(B_{0}\right) \supset C_{0}^{\infty}(V \times S)$ and from the explicit expression for the resolvent operator

$$
\left(\left(\lambda I-B_{0}\right)^{-1} \psi\right)(\mathbf{x}, \mathbf{u})=\frac{1}{c} \int_{0}^{R(\mathbf{x}, \mathbf{u})} \exp (-\lambda r / c) \psi(\mathbf{x}-r \mathbf{u}, \mathbf{u}) d r
$$

where $R(\mathbf{x}, \mathbf{u})$ is such that $\mathbf{x}-R(\mathbf{x}, \mathbf{u}) \mathbf{u} \in \Sigma$ ( and we assume that $R(\mathbf{x}, \mathbf{u})$ is a continuous function of $(\mathbf{x}, \mathbf{u}) \in V \times S)$.

Relation (2.15) implies that

$$
\left\|\left(\lambda I-B_{0}\right)^{-1}\right\|_{B(X)} \leq 1 / \lambda \quad \forall \lambda>0 .
$$

Further, the semigroup generated by $B_{0}$ has the form

$$
\left(\exp \left(t B_{0}\right) \psi\right)(\mathbf{x}, \mathbf{u})=\psi(\mathbf{x}-c t \mathbf{u}, \mathbf{u}) \chi_{V}(\mathbf{x}-c t \mathbf{u}), \quad \forall \psi \in X, t \geq 0,
$$

where $\chi_{V}$ is the characteristic function of $V$.

(c) $B \in \mathcal{G}\left(1,-c \sigma_{\min } ; X\right)$, i.e. the streaming operator with absorption, $\mathrm{B}$, is the infinitesimal generator of the contraction semigroup $\{\exp (t B)\}_{t \geq 0}$. This follows from the fact that $D(B)=D\left(B_{0}\right)$ and from the expression for the resolvent operator

$$
\left((\lambda I-B)^{-1} \psi\right)(\mathbf{x}, \mathbf{u})=\frac{1}{c} \int_{0}^{R(\mathbf{x}, \mathbf{u})} \exp \left\{-\frac{1}{c} \int_{0}^{r}\left[\lambda+c \sigma\left(\mathbf{x}-r^{\prime} \mathbf{u}\right)\right] d r^{\prime}\right\} \psi(\mathbf{x}-r \mathbf{u}, \mathbf{u}) d r
$$

which implies that

$$
\left\|(\lambda I-B)^{-1}\right\|_{B(X)} \leq 1 /\left(\lambda+c \sigma_{\min }\right) \quad \forall \lambda>-c \sigma_{\min } .
$$

The semigroup generated by $B$ has the form

$$
(\exp (t B) \psi)(\mathbf{x}, \mathbf{u})=\exp \left[-\int_{0}^{c t} \sigma(\mathbf{x}-r \mathbf{u}) d r\right] \psi(\mathbf{x}-c t \mathbf{u}, \mathbf{u}) \chi_{V}(\mathbf{x}-c t \mathbf{u}), \forall \psi \in X, t \geq 0 .
$$

(d) $T \in \mathcal{G}\left(1,-c\left(\sigma_{\min }-\sigma_{s, \max }\right) ; X\right)$, i.e $T$ is the infinitesimal generator of the $C_{0}$-semigroup $\{\exp (t T)\}_{t \geq 0}$. This follows since $D(T)=D\left(B_{0}\right)$ and

$$
(\lambda I-T)^{-1}=\left(I-(\lambda I-B)^{-1} K\right)^{-1}(\lambda I-B)^{-1},
$$

from which we can deduce that

$$
\left\|(\lambda I-T)^{-1}\right\|_{B(X)} \leq 1 /\left[\lambda+c\left(\sigma_{\min }-\sigma_{s, \max }\right)\right], \quad \forall \lambda>-c\left(\sigma_{\min }-\sigma_{s, \max }\right) .
$$

We remark that, if the cloud is homogeneous, then $\sigma(\mathbf{x})=\sigma$ and $\sigma_{s}(\mathbf{x})=\sigma_{s}$, where $\sigma$ and $\sigma_{s}$ are constants. Also, $\sigma=\sigma_{c}+\sigma_{s}$ where $\sigma_{c}>0$ is the capture cross-section. Hence, in the homogeneous case, $\sigma_{\min }=\sigma, \sigma_{s, \max }=\sigma_{s}$ and $\sigma_{\min }-\sigma_{s, \max }=\sigma-\sigma_{s}=\sigma_{c}>0$. 
By using definitions $(2.7)-(2.10)$, the abstract version of the system $(2.1)-(2.3)$ can be written as

$$
\frac{d}{d t} N(t)=T[N(t)]+q(t), \quad t>0 ; \quad N(0)=N_{0}
$$

where now $N(t)=N(\cdot, \cdot, t)$ and $q(t)=q(\cdot, \cdot, t))$ are maps from $[0, \infty)$ into $X$ and $N_{0}$ is a given element in $X$. Due to result $(\mathrm{d})$, the unique solution of $(2.23)$ has the form

$$
N(t)=\exp (t T) N_{0}+\int_{0}^{t} \exp ((t-s) T) q(s) d s, \quad t \geq 0,
$$

provided that $N_{0} \in D(T)$ and $q(t)$ is strongly continuously differentiable; see [1].

Remark 2.1 If $N_{1}(t)$ is the solution of (2.23) with $q(t)$ replaced by $q_{1}(t)$, we have from (2.24)

$$
\begin{aligned}
\left\|N_{1}(t)-N(t)\right\|_{1} & \leq \int_{0}^{t} \exp \left[-c\left(\sigma_{\min }-\sigma_{s, \max }\right)(t-s)\right]\left\|q_{1}(s)-q(s)\right\|_{1} d s \\
& \leq \frac{1}{c\left(\sigma_{\min }-\sigma_{s, \max }\right)}\left\{1-\exp \left[-c\left(\sigma_{\min }-\sigma_{s, \max }\right) \bar{t}\right]\right\} \max _{s \in[0, \bar{t}]}\left\|q_{1}(s)-q(s)\right\|_{1}, \forall t \in[0, \bar{t}] .
\end{aligned}
$$

Hence the solution to our evolution problem depends continuously on the source. Further, if in particular $q(\mathbf{x}, \mathbf{u}, s)=\phi(s) \Phi(\mathbf{x}, \mathbf{u})$ and $q_{1}(\mathbf{x}, \mathbf{u}, s)=\phi(s) \Phi_{1}(\mathbf{x}, \mathbf{u})$, we have

$$
\left\|q_{1}(s)-q(s)\right\|_{1} \leq\left\|\Phi_{1}-\Phi\right\|_{1} \max _{s \in[0, \bar{t}]}|\phi(s)|
$$

Note that (2.24) cannot be used if the source has the form $q_{0}(t) \delta\left(\mathbf{x}-\mathbf{x}_{0}\right)$ (with $\mathbf{x}_{0} \in V$ and $q_{0}(t)$ continuously differentiable), because $\delta\left(\mathbf{x}-\mathbf{x}_{0}\right)$ is not an element of the Banach space $X$. Instead, we must find an appropriate space of test functions and corresponding generalized functions on $V \times S$, say $Z(=Z(V \times S))$ and $Z^{\prime}(=$ $\left.Z^{\prime}(V \times S)\right)$ respectively. As will become apparent, three essential requirements of these spaces are the following. Firstly, $Z^{\prime}$ must contain the localised photon source, which strictly must be interpreted as the direct product $q_{0}(t) \mathbf{1}(\mathbf{u}) \otimes \delta\left(\mathbf{x}-\mathbf{x}_{0}\right)$ defined by

$$
\left\langle q_{0}(t) \mathbf{1}(\mathbf{u}) \otimes \delta\left(\mathbf{x}-\mathbf{x}_{0}\right), \xi\right\rangle:=q_{0}(t) \int_{S} \xi\left(\mathbf{x}_{0}, \mathbf{u}\right) d \mathbf{u}, \quad \forall \xi \in Z,
$$

where $\langle f, \psi\rangle$ denotes the action of $f \in Z^{\prime}$ on $\psi \in Z$. Secondly, each function $\psi \in X$ must generate a regular generalized function, $\tilde{\psi} \in Z^{\prime}$, via

$$
\langle\tilde{\psi}, \xi\rangle:=\int_{S} \int_{V} \psi(\mathbf{x}, \mathbf{u}) \xi(\mathbf{x}, \mathbf{u}) d \mathbf{x} d \mathbf{u} \quad \forall \xi \in Z,
$$

and therefore, since the set $\tilde{X}$ of all regular generalized functions $\tilde{\psi}$ generated by $\psi \in X$ lies in $Z^{\prime}$, there is a natural embedding of $X$ in $Z^{\prime}$. Thirdly, an appropriately defined "extension", $\tilde{T}$, of the operator $T$ from $D(T) \subseteq X$ to $Z^{\prime}$ must exist satisfying

$$
\tilde{T} \tilde{\psi}=\widetilde{T \psi} \quad \forall \psi \in D(T)
$$

Note that this requires $\widetilde{D(T)}$ to be a subspace of $D(\tilde{T})$, where

$$
\widetilde{D(T)}=\left\{\tilde{\psi} \in Z^{\prime}: \psi \in D(T)\right\} .
$$


Equation (2.23) can then be extended to the generalized problem

$$
\frac{d}{d t} N(t)=\tilde{T}[N(t)]+q_{0}(t) \mathbf{1}(\mathbf{u}) \otimes \delta\left(\mathbf{x}-\mathbf{x}_{0}\right) ; \quad N(0)=N_{0},
$$

where a solution $N:[0, \infty) \rightarrow Z^{\prime}$ is now sought.

\section{The Adjoint Transport Semigroup}

In this section our aim is to produce an operator, say $A$, defined in some Banach space $Y$ of continuous functions on $V \times S$ such that $A$ generates a $C_{0}$-semigroup on $Y$ and is adjoint to the operator $T$ of the previous section in the sense that

$$
\int_{S} \int_{V}(A \eta)(\mathbf{x}, \mathbf{u}) \psi(\mathbf{x}, \mathbf{u}) d \mathbf{x} d \mathbf{u}=\int_{S} \int_{V} \eta(\mathbf{x}, \mathbf{u})(T \psi)(\mathbf{x}, \mathbf{u}) d \mathbf{x} d \mathbf{u}, \quad \forall \eta \in D(A) \subset Y, \psi \in D(T) \subset X .
$$

Largely due to the fact that $X$ is not a reflexive Banach space, the approach we use is rather technical and involves the theory of sun dual semigroups [5, pp. 62-63] and a perturbation theorem due to Suhadolc and Vidav [15, Lemma 2.2]. For clarity, we shall split the following manipulations into six parts.

(I) In the usual manner, the dual space $X^{*}$ can be identified with $L^{\infty}(V \times S)$, with norm

$$
\|\eta\|_{\infty}=\operatorname{ess}_{(\mathbf{x}, \mathbf{u}) \in V \times S}|\eta(\mathbf{x}, \mathbf{u})|,
$$

via the duality pairing

$$
(\eta, \psi)=\int_{S} \int_{V} \eta(\mathbf{x}, \mathbf{u}) \psi(\mathbf{x}, \mathbf{u}) d \mathbf{x} d \mathbf{u}, \quad \psi \in X .
$$

The adjoint operator $B^{*}$ is then defined by

$$
\left(B^{*} \eta\right)(\mathbf{x}, \mathbf{u})=c \mathbf{u} \cdot \nabla_{\mathbf{x}} \eta(\mathbf{x}, \mathbf{u})-c \sigma(\mathbf{x}) \eta(\mathbf{x}, \mathbf{u})
$$

with domain

$$
D\left(B^{*}\right)=\left\{\eta \in X^{*}: c \mathbf{u} \cdot \nabla_{\mathbf{x}} \eta \in X^{*}, \eta(\mathbf{y}, \mathbf{u})=0 \text { if } \mathbf{y} \in \Sigma \text { and } \mathbf{u} \cdot \mathbf{n}(\mathbf{y})>0\right\} .
$$

Note that $D\left(B^{*}\right)$ is not dense in $X^{*}$ and so $B^{*}$ cannot be the generator of a $C_{0}$-semigroup on $X^{*}$.

Let the family of operators $\{W(t)\}_{t \geq 0}$ be defined on $X^{*}$ by

$$
[W(t) \eta](\mathbf{x}, \mathbf{u})=\exp \left[-\int_{0}^{c t} \sigma(\mathbf{x}+r \mathbf{u}) d r\right] \eta(\mathbf{x}+c t \mathbf{u}, \mathbf{u}) \chi_{V}(\mathbf{x}+c t \mathbf{u}) .
$$


Then, for any $\psi \in X$ and $\eta \in X^{*}$, we have

$$
\begin{aligned}
& (\eta, \exp (t B) \psi) \\
= & \int_{S} \int_{V} \eta(\mathbf{x}, \mathbf{u}) \exp \left[-\int_{0}^{c t} \sigma(\mathbf{x}-r \mathbf{u}) d r\right] \psi(\mathbf{x}-c t \mathbf{u}, \mathbf{u}) \chi_{V}(\mathbf{x}-c t \mathbf{u}) d \mathbf{x} d \mathbf{u} \\
= & \int_{S} \int_{\mathbb{R}^{3}} \eta(\mathbf{x}, \mathbf{u}) \exp \left[-\int_{0}^{c t} \sigma(\mathbf{x}-r \mathbf{u}) d r\right] \psi(\mathbf{x}-c t \mathbf{u}, \mathbf{u}) \chi_{V}(\mathbf{x}-c t \mathbf{u}) \chi_{V}(\mathbf{x}) d \mathbf{x} d \mathbf{u} \\
= & \int_{S} \int_{\mathbb{R}^{3}} \eta(\mathbf{z}+c t \mathbf{u}, \mathbf{u}) \exp \left[-\int_{0}^{c t} \sigma(\mathbf{z}+c t \mathbf{u}-r \mathbf{u}) d r\right] \psi(\mathbf{z}, \mathbf{u}) \chi_{V}(\mathbf{z}) \chi_{V}(\mathbf{z}+c t \mathbf{u}) d \mathbf{z} d \mathbf{u} \\
= & \int_{S} \int_{V} \exp \left[-\int_{0}^{c t} \sigma\left(\mathbf{z}+r^{\prime} \mathbf{u}\right) d r^{\prime}\right] \eta(\mathbf{z}+c t \mathbf{u}, \mathbf{u}) \chi_{V}(\mathbf{z}+c t \mathbf{u}) \psi(\mathbf{z}, \mathbf{u}) d \mathbf{z} d \mathbf{u} \\
= & (W(t) \eta, \psi) .
\end{aligned}
$$

Consequently,

$$
W(t)=(\exp (t B))^{*}, \forall t \geq 0
$$

(II) Standard duality arguments show that the algebraic semigroup properties of $\left\{e^{t B}\right\}_{t \geq 0}$ on $X$ are inherited by $\{W(t)\}_{t \geq 0}=\left\{(\exp (t B))^{*}\right\}_{t \geq 0}$ on $X^{*}$. However, to obtain a strongly continuous adjoint semigroup we must restrict each operator $W(t)$ to the sun dual (or semigroup dual) $X^{\odot} \subset X^{*}$ of $X$ defined by

$$
X^{\odot}:=\left\{\eta \in X^{*}: \lim _{t \downarrow 0}\|W(t) \eta-\eta\|_{X^{*}}=0\right\} .
$$

As discussed in [5, pp.62-63], the space $X^{\odot}=\overline{D\left(B^{*}\right)}$ and so $X^{\odot}$ is a closed subspace of $X^{*}$. Moreover, if we denote the restriction of $W(t)$ to $X^{\odot}$ by $e^{t B^{\odot}}$, then $\left\{e^{t B^{\odot}}\right\}_{t \geq 0}$ (the sun dual semigroup of $\left\{e^{t B}\right\}_{t \geq 0}$ ) is a $C_{0}$-semigroup on $X^{\odot}$ and has generator $\left(B^{\odot}, D\left(B^{\odot}\right)\right)$ defined by

$$
B^{\odot} \eta=B^{*} \eta, \quad D\left(B^{\odot}\right)=\left\{\eta \in D\left(B^{*}\right): B^{*} \eta \in X^{\odot}\right\}
$$

In addition,

$$
\left\|e^{t B^{\odot}} \eta\right\|_{X^{*}} \leq e^{-c t \sigma_{\min }}\|\eta\|_{X^{*}} \forall \eta \in X^{\odot} \text { and } t \geq 0
$$

(III) Motivated by [15, eqn.(2b)], we now define a closed subspace $Y$ of $X^{\odot}$ by

$$
Y:=\{\eta \in C(V \times S): \eta(\mathbf{y}, \mathbf{u})=0 \text { if } \mathbf{y} \in \Sigma \text { and } \mathbf{u} \cdot \mathbf{n}(\mathbf{y}) \geq 0\}
$$

and denote the restriction of $e^{t B^{\odot}}$ to $Y$ by $e^{t G}$. Note that, from (3.5), when $t$ is sufficiently small we have

$$
[W(t) \eta](\mathbf{x}, \mathbf{u})= \begin{cases}\exp \left[-\int_{0}^{c t} \sigma(\mathbf{x}+r \mathbf{u}) d r\right] \eta(\mathbf{x}+c t \mathbf{u}) & \text { when } \mathbf{x} \in V_{i}, \mathbf{u} \in S \\ \exp \left[-\int_{0}^{c t} \sigma(\mathbf{x}+r \mathbf{u}) d r\right] \eta(\mathbf{x}+c t \mathbf{u}) & \text { when } \mathbf{x} \in \Sigma, \mathbf{u} \in S: \mathbf{u} \cdot \mathbf{n}(\mathbf{x})<0 \\ 0 & \text { when } \mathbf{x} \in \Sigma, \mathbf{u} \in S: \mathbf{u} \cdot \mathbf{n}(\mathbf{x}) \geq 0\end{cases}
$$

Consequently, $\lim _{t \downarrow 0}\|W(t) \eta-\eta\|_{X^{*}}=0$ for all $\eta \in Y$ and so $Y$ is a closed subspace of $X^{\odot}$. Moreover, for each $t>0$ and $\eta \in Y,[W(t) \eta](\mathbf{x}, \mathbf{u})=0$ whenever $\mathbf{x} \in \Sigma$ and $\mathbf{u} \cdot \mathbf{n}(\mathbf{x}) \geq 0$. Also, if $\left(\mathbf{x}_{m}, \mathbf{u}_{m}\right) \rightarrow(\mathbf{x}, \mathbf{u})$ in $V \times S$ as $m \rightarrow \infty$ then, on using the continuity of $\sigma,[W(t) \eta]\left(\mathbf{x}_{m}, \mathbf{u}_{m}\right) \rightarrow[W(t) \eta](\mathbf{x}, \mathbf{u})$. Consequently, 
$W(t) Y \subset Y$ for each $t \geq 0$ and it follows from [5, pp.60-61] that $\left\{e^{t G}\right\}_{t \geq 0}$ is a $C_{0}$-semigroup on $Y$ and also that the generator $G$ of $\left\{e^{t G}\right\}_{t \geq 0}$ is given by

$$
G \eta=B^{\odot} \eta, \quad D(G)=\left\{\eta \in D\left(B^{\odot}\right) \cap Y: B^{\odot} \eta \in Y\right\} \subset Y .
$$

As $G$ is a restriction of $B^{*}$, it follows that $G$ is adjoint to $B$. Moreover, from (3.7),

$$
\left\|e^{t G} \eta\right\|_{X^{*}} \leq e^{-c t \sigma_{m i n}}\|\eta\|_{X^{*}} \quad \forall \eta \in Y \text { and } t \geq 0 .
$$

(IV) We now examine the effect of the operator $K \in B(X)$ defined by (2.7). The adjoint operator $K^{*}$, given by

$$
\left(K^{*} \eta\right)(\mathbf{x}, \mathbf{u})=c \sigma_{s}(\mathbf{x}) \int_{S} k\left(\mathbf{u}, \mathbf{u}^{\prime}\right) \eta\left(\mathbf{x}, \mathbf{u}^{\prime}\right) d \mathbf{u}^{\prime},
$$

is a bounded operator on $X^{*}$ and satisfies

$$
\left\|K^{*}\right\|_{B\left(X^{*}\right)} \leq c \sigma_{s, \max } .
$$

Bearing in mind (3.8), we suppose initially, that the scattering cross-section $\sigma_{s}$ is zero on the boundary $\Sigma$ of $V$; this is reasonable from a physical viewpoint because the cloud particle density approaches zero as $\mathbf{x}$ tends to the boundary. In this case, the restriction $K_{\left.\right|_{Y}}^{*}$ of $K^{*}$ to $Y$ is a bounded operator on $Y$ and, since $\left\|K_{\left.\right|_{Y}}^{*} \eta\right\|_{X^{*}} \leq c \sigma_{s, \max }\|\eta\|_{X^{*}}$ for all $\eta \in Y$, it follows that $(A, D(A))$, where $A=G+K_{\left.\right|_{Y}}^{*}$ and $D(A)=D(G)$, is the infinitesimal generator of a $C_{0}$-semigroup $\left\{e^{t A}\right\}_{t \geq 0}$ on $Y$ which satisfies $\left\|e^{t A} \eta\right\|_{X^{*}} \leq e^{-c t\left(\sigma_{\min }-\sigma_{s, \max }\right)}\|\eta\|_{X^{*}}$ for all $\eta \in Y$ and $t \geq 0$. In addition $A$ satisfies the equation

$$
\begin{aligned}
(A \eta, \psi) & =\left(G \eta+K_{\left.\right|_{Y}}^{*} \eta, \psi\right) \\
& =\left(B^{*} \eta+K_{\left.\right|_{Y}}^{*} \eta, \psi\right) \\
& =(\eta, B \psi+K \psi)=(\eta, T \psi) \forall \eta \in D(A), \psi \in D(T) .
\end{aligned}
$$

Thus, the operator $A$ is adjoint to $T$.

(V) The situation is more complicated when $\sigma_{s}$ is not identically zero on the boundary $\Sigma$ since then the operator $K_{\left.\right|_{Y}}^{*}$ does not map $Y$ into $Y$. Note that this case is mainly of mathematical interest. To deal with scattering cross-sections of this type, we use the following result [15, Lemma 2.2].

Lemma 3.1 (Suhadolc and Vidav) Let $\mathcal{U}$ be a closed subspace of a Banach space $\mathcal{V}$ and assume that each of the following conditions holds.

(i) The operator $\mathcal{P}$ is the infinitesimal generator of a $C_{0}$-semigroup $\{S(t)\}_{t \geq 0} \in \mathcal{G}(1, \omega ; \mathcal{U})$.

(ii) There exists a densely-defined operator $P: \mathcal{U} \supset D(P) \rightarrow \mathcal{V}$, where $D(\mathcal{P}) \subset D(P)$, such that $\mathcal{P} \phi=P \phi$ for all $\phi \in D(\mathcal{P})$. Thus, $P$ is an extension of $\mathcal{P}$.

(iii) $(\lambda I-P)^{-1} \in B(\mathcal{V}, \mathcal{U})$ for all $\lambda$ with $\Re \lambda>\omega$ (where $I$ denotes the inclusion map from $\mathcal{U}$ into $\mathcal{V}$ ) and

$$
\left\|(\lambda I-P)^{-1}\right\|_{B(\mathcal{V}, \mathcal{U})} \leq 1 /[\Re \lambda-\omega] \text { for } \Re \lambda>\omega .
$$


(iv) $Q$ is any given operator that belongs to $B(\mathcal{U}, \mathcal{V})$.

Then there exists a dense subspace $\mathcal{D}$ of $\mathcal{U}$ such that the restriction $(P+Q)_{\left.\right|_{\mathcal{D}}}$ of $P+Q$ to $\mathcal{D}$ is the infinitesimal generator of a $C_{0}$-semigroup of operators on $\mathcal{U}$.

Proof See [15, pp. 518-519].

To apply Lemma 3.1 to our problem, we take $\mathcal{V}=C(V \times S)$ (equipped with the usual supremum norm), $\mathcal{U}=Y, \mathcal{P}=G$ and $Q=K^{*}$ where $Y, G$ and $K^{*}$ are defined by (3.8), (3.9) and (3.11) respectively. Then (i) and (iv) of Lemma 3.1 clearly hold with $\omega=-c \sigma_{\min }$ and $\left\|K^{*} \eta\right\|_{\infty} \leq c \sigma_{s, \max }\|\eta\|_{\infty}$ for all $\eta \in Y$. It remains to show that (ii) and (iii) are also satisfied. To do this we adapt the argument used to prove

[15, Lemma 2.3]. First note that for $\Re \lambda>-c \sigma_{\text {min }}$,

$$
\begin{aligned}
& {\left[(\lambda I-B)^{-1} \psi\right](\mathbf{x}, \mathbf{u}) }=\int_{0}^{\infty}\left(e^{t B} \psi\right)(\mathbf{x}, \mathbf{u}) e^{-\lambda t} d t \\
&=\int_{0}^{\infty} \exp \left[-\lambda t-\int_{0}^{c t} \sigma(\mathbf{x}-r \mathbf{u}) d r\right] \psi(\mathbf{x}-c t \mathbf{u}, \mathbf{u}) \chi_{V}(\mathbf{x}-c t \mathbf{u}) d t \\
& \text { where } \psi \in X \supset C(V \times S) .
\end{aligned}
$$

It follows that

$$
\begin{gathered}
\left(\left[(\lambda I-B)^{-1}\right]^{*} \eta\right)(\mathbf{x}, \mathbf{u})=\int_{0}^{\infty} \exp \left[-\lambda t-\int_{0}^{c t} \sigma(\mathbf{x}-r \mathbf{u}) d r\right] \eta(\mathbf{x}+c t \mathbf{u}, \mathbf{u}) \chi_{V}(\mathbf{x}+c t \mathbf{u}) d t \\
\text { where } \eta \in X^{*} \supset C(V \times S) .
\end{gathered}
$$

Lemma 3.2 Let $R(\lambda)$ be defined by

$$
R(\lambda)=\left[(\lambda I-B)^{-1}\right]_{\left.\right|_{C(V \times S)}}^{*}=\left(\lambda I-B^{*}\right)_{\left.\right|_{C(V \times S)}}^{-1}, \Re \lambda>-c \sigma_{\min } .
$$

Then

(i) $R(\lambda)$ maps $C(V \times S)$ into $Y$ and $R(\lambda)_{\left.\right|_{Y}}=(\lambda I-G)^{-1}$;

(ii) $\|R(\lambda) \eta\|_{\infty} \leq\|\eta\|_{\infty} /\left[\Re \lambda+c \sigma_{\min }\right]$ and $R(\lambda)$ is one-to-one;

(iii) $R\left(\lambda_{1}\right) R\left(\lambda_{2}\right)=R\left(\lambda_{2}\right) R\left(\lambda_{1}\right)$ and $R\left(\lambda_{2}\right)-R\left(\lambda_{1}\right)=\left(\lambda_{1}-\lambda_{2}\right) R\left(\lambda_{1}\right) R\left(\lambda_{2}\right)$ for $\Re \lambda_{i}>-c \sigma_{\text {min }}, i=1,2$.

Proof Parts (ii) and (iii) follow automatically from the definition of $R(\lambda)$. For (i), we note that $R(\lambda) \eta \in C(V \times S)$ for each $\eta \in C(V \times S)$. Moreover, if $\mathbf{x} \in \Sigma$ and $\mathbf{u}$ is outgoing then $\eta(\mathbf{x}+\operatorname{ct} \mathbf{u}, \mathbf{u})=0$ for all $t \geq 0$ and so $R(\lambda) \eta \in Y$. Finally,

$$
(\lambda I-G)^{-1} \eta=\int_{0}^{\infty} e^{-\lambda t} e^{t G} \eta d t=\int_{0}^{\infty} e^{-\lambda t} W(t) \eta d t=R(\lambda) \eta, \forall \eta \in Y .
$$

(VI) We now define the operator $\hat{G}$ by

$$
\hat{G} \eta=\left(\lambda I-[R(\lambda)]^{-1}\right) \eta, \eta \in D(\hat{G})=\operatorname{Range}(R(\lambda)) \subset Y,
$$


Since

$$
\hat{G} \eta=\lambda \eta-\left(\lambda \eta-B^{*} \eta\right), \eta \in D(\hat{G})
$$

it follows that $\hat{G}$ is a restriction of $B^{*}$ to $D(\hat{G})$. Similarly, $B^{*}$ is also an extension of $G$ since

$$
R(\lambda)(\lambda I-G) \eta=(\lambda I-G)^{-1}(\lambda I-G) \eta=\eta, \forall \eta \in D(G)
$$

Moreover,

$$
\lambda I-\hat{G}=[R(\lambda)]^{-1} \Rightarrow(\lambda I-\hat{G})^{-1}=R(\lambda)
$$

and so $\left\|(\lambda I-\hat{G})^{-1} \eta\right\|_{\infty} \leq\|\eta\|_{\infty} /\left[\Re \lambda+c \sigma_{m i n}\right]$. Therefore the conditions of Lemma 3.1 all hold with $P=\hat{G}$. This establishes the existence of a dense subspace $\mathcal{D}$ of $Y$ such that

$$
A=\left(\hat{G}+K^{*}\right)_{\left.\right|_{\mathcal{D}}}
$$

is the infinitesimal generator of a $C_{0}$-semigroup of operators $\{\exp (t A)\}_{t \geq 0}$ on $Y$, with

$$
\left\|e^{t A} \eta\right\|_{\infty} \leq e^{-c t\left(\sigma_{\min }-\sigma_{s, \max }\right)}\|\eta\|_{\infty} \text { for all } \eta \in Y \text { and } t \geq 0
$$

and

$$
\left\|(\lambda I-A)^{-1}\right\| \leq 1 /\left[\lambda+c\left(\sigma_{\min }-\sigma_{s, \max }\right)\right], \quad \forall \lambda>-c\left(\sigma_{\min }-\sigma_{s, \max }\right) .
$$

In addition, $A$ satisfies the equation

$$
\begin{aligned}
(A \eta, \psi) & =\left(\left(B^{*}+K^{*}\right)_{\left.\right|_{\mathcal{D}}} \eta, \psi\right) \\
& =\left(\left(B^{*}+K^{*}\right) \eta, \psi\right) \\
& =(\eta, B \psi+K \psi)=(\eta, T \psi), \quad \forall \eta \in D(A), \psi \in D(T) .
\end{aligned}
$$

Summarising, the key properties of $A$ established above, for both $\sigma_{s} \equiv 0$ on $\Sigma$ and $\sigma_{s} \not \equiv 0$ on $\Sigma$ are

$$
\begin{gathered}
A \in \mathcal{G}\left(1,-c\left(\sigma_{\min }-\sigma_{s, \max }\right) ; Y\right), \\
(\lambda I-A)^{-1} \in B(Y) \text { for all } \lambda>-c\left(\sigma_{\min }-\sigma_{s, \max }\right),
\end{gathered}
$$

and

$$
(A \eta, \psi)=(\eta, T \psi), \quad \forall \eta \in D(A), \psi \in D(T)
$$

\section{Semigroups on Countably Normed Spaces}

Throughout this section, $\mathcal{X}$ will represent a Fréchet space in which the topology is generated by a countable collection of norms $\left\{|\cdot|_{n}\right\}_{n=0}^{\infty}$ where

$$
|\phi|_{0} \leq|\phi|_{1} \leq \cdots \leq|\phi|_{k} \leq \cdots \quad \forall \phi \in \mathcal{X}
$$


see $[7$, p.15]. We shall also assume that there exists a nested chain of Banach spaces

$$
\left(\mathcal{X}_{0},|\cdot|_{0}\right) \supset\left(\mathcal{X}_{1},|\cdot|_{1}\right) \supset \cdots \supset\left(\mathcal{X}_{k},|\cdot|_{k}\right) \supset \cdots
$$

such that, for each $k, \mathcal{X} \subset \mathcal{X}_{k}$ and

$$
\overline{\left(\mathcal{X},|\cdot|_{k}\right)}=\left(\mathcal{X}_{k},|\cdot|_{k}\right) .
$$

It follows that $\mathcal{X}$ may be regarded as both a complete countably multinormed space ([19, Section 1.6]) and a complete countably normed space ([7, p.16]). The completeness of $\mathcal{X}$ also means that

$$
\mathcal{X}=\bigcap_{k=0}^{\infty} \mathcal{X}_{k}
$$

see $[7$, p.17]. We shall denote by $L(\mathcal{X})$ the vector space of continuous linear operators mapping $\mathcal{X}$ into $\mathcal{X}$. A family $F$ of operators in $L(\mathcal{X})$ is said to be equicontinuous if for any continuous seminorm $\gamma$ on $\mathcal{X}$ there exists a continuous seminorm $\rho$ on $\mathcal{X}$ ( $\rho$ depending on $\gamma$ ) such that $\gamma(\mathcal{B} \phi) \leq \rho(\phi) \forall \mathcal{B} \in F$ and $\phi \in \mathcal{X}$.

\section{Remark 4.1}

(i) Note that if $\gamma$ is a continuous seminorm on $\mathcal{X}$, then a simple modification of the proof of [19, Theorem 1.8.1] shows that

$$
\gamma(\phi) \leq C \max \left\{|\phi|_{0},|\phi|_{1}, \ldots,|\phi|_{r}\right\},
$$

where $C$ and $r$ depend on $\gamma$ but not on $\phi$. Consequently, to verify that the family $F$ is equicontinuous we need only establish that for each $k=0,1, \ldots$, there exists a continuous seminorm $\rho_{k}$ on $\mathcal{X}$ such that

$$
|\mathcal{B} \phi|_{k} \leq \rho_{k}(\phi) \quad \forall \mathcal{B} \in F \text { and } \phi \in \mathcal{X}
$$

(ii) Strictly, the definition of a countably normed space in [7] requires the family of norms $\left\{|\cdot|_{k}\right\}$ to be compatible.

However, this condition is only imposed so that, for each $k$, the completion of $\mathcal{X}$ with respect to the norm $|\cdot|_{k+1}$ is mapped in a one-to-one manner into the completion of $\mathcal{X}$ with respect to $|\cdot|_{k}$; see [7, pp. 12-13]. Our assumptions (4.2) and (4.3) allow us to ignore this compatibility condition.

Let $\mathcal{A}$ be the infinitesimal generator of an equicontinuous semigroup $\{\exp (t \mathcal{A})\}_{t \geq 0}$ on $\mathcal{X}$ of class $C_{0}$ (see $[18$, Chapter IX], and consider the abstract Cauchy problem

$$
\frac{d}{d t} u(t)=\mathcal{A}[u(t)]+q(t) \quad(t>0) ; \quad u(0)=u_{0},
$$

where $u_{0}$ is a given element in $\mathcal{X}, q \in C^{1}([0, \infty), \mathcal{X})$ and the derivative is defined in terms of the topology on $\mathcal{X}$. As our approach for dealing with the distributional photon transport problem is designed to yield an infinitesimal generator that is continuous and everywhere defined on an appropriate Fréchet space of test functions (see $\S 5$ ), we shall, for simplicity, now assume that $\mathcal{A} \in L(\mathcal{X})$. In this case, routine modifications to the usual Banach space arguments show that (4.6) has a unique solution $u \in C([0, \infty), \mathcal{X}) \cap C^{1}((0, \infty), \mathcal{X})$ given by

$$
u(t)=e^{t \mathcal{A}} u_{0}+\int_{0}^{t} e^{(t-s) \mathcal{A}} q(s) d s
$$


We now consider ACPs posed in the dual space $\mathcal{X}^{\prime}$ of $\mathcal{X}$. We shall always assume that $\mathcal{X}^{\prime}$ is equipped with the weak*-topology and is therefore sequentially complete. The action of a functional $f \in \mathcal{X}^{\prime}$ on an element $\phi \in \mathcal{X}$ will be written as $\langle f, \phi\rangle$. Moreover, the operator $\mathcal{A}^{\prime}$ will denote the adjoint of the infinitesimal generator $\mathcal{A}$ of the equicontinuous semigroup $\left\{e^{t \mathcal{A}}\right\}_{t \geq 0}$. Thus,

$$
\left\langle\mathcal{A}^{\prime} f, \phi\right\rangle=\langle f, \mathcal{A} \phi\rangle, \quad \forall f \in \mathcal{X}^{\prime} \text { and } \phi \in \mathcal{X}
$$

The ACP we examine is

$$
\frac{d}{d t} u(t)=\mathcal{A}^{\prime}[u(t)]+q(t) \quad(t>0) ; \quad u(0)=u_{0},
$$

where $u_{0}$ is a given element in $\mathcal{X}^{\prime}, q$ is a function from $[0, \infty)$ into $\mathcal{X}^{\prime}$ and the derivative is defined in terms of the weak*-topology.

Theorem 4.2 (Existence and Uniqueness Results in $\mathcal{X}^{\prime}$.)

The unique solution $u \in C\left([0, \infty), \mathcal{X}^{\prime}\right) \cap C^{1}\left((0, \infty), \mathcal{X}^{\prime}\right)$ of $(4.8)$ is given by

$$
u(t)=e^{t \mathcal{A}^{\prime}} u_{0}+\int_{0}^{t} e^{(t-s) \mathcal{A}^{\prime}} q(s) d s,
$$

where the integral exists as a Riemann integral that is weak*-convergent in $\mathcal{X}^{\prime}$.

Proof That (4.9) is a solution of (4.8) follows by direct differentiation. To show that (4.8) defines the only solution in $C\left([0, \infty), \mathcal{X}^{\prime}\right) \cap C^{1}\left((0, \infty), \mathcal{X}^{\prime}\right)$ we need only verify that the unique solution of the homogeneous problem is $u(t)=\exp \left(t \mathcal{A}^{\prime}\right) u_{0}$. Suppose that $v \in C\left([0, \infty), \mathcal{X}^{\prime}\right) \cap C^{1}\left((0, \infty), \mathcal{X}^{\prime}\right)$ satisfies $(4.8)$ with $q \equiv 0$. Then, for any $\phi \in \mathcal{X}$ and $0 \leq s \leq t<\infty$,

$$
\begin{aligned}
& \frac{d}{d s}\left\langle e^{(t-s) \mathcal{A}^{\prime}} u(s), \phi\right\rangle=\frac{d}{d s}\left\langle u(s), e^{(t-s) \mathcal{A}} \phi\right\rangle \\
= & \left\langle\frac{d}{d s} u(s), e^{(t-s) \mathcal{A}} \phi\right\rangle-\left\langle u(s), \mathcal{A} e^{(t-s) \mathcal{A}} \phi\right\rangle \quad \text { (by [7, p.72]) } \\
= & \left\langle\mathcal{A}^{\prime} u(s), e^{(t-s) \mathcal{A}} \phi\right\rangle-\left\langle\mathcal{A}^{\prime} u(s), e^{(t-s) \mathcal{A}} \phi\right\rangle=0,
\end{aligned}
$$

and so $v(t)=\exp \left(t \mathcal{A}^{\prime}\right) u_{0}$ as required.

\section{The space $Z^{\prime}$ of generalized functions}

To obtain a space $Z^{\prime}$ possessing the properties described in Section 2, we follow the approach used in [2, Section 3.2]. Let $Y$ be the Banach space given by (3.8), let $A$ satisfy (3.15) - (3.17), and define a space of test functions by

$$
Z:=\bigcap_{k=0}^{\infty} D\left(A^{k}\right)(\subset Y)
$$

where $A^{0}$ is interpreted as the identity operator. As in [2], the topology in $Z$ is generated by the countable collection of seminorms $\left\{\alpha_{k}\right\}_{k=0}^{\infty}$ where $\alpha_{k}(\xi):=\left\|A^{k} \xi\right\|_{Y}, \xi \in Z$. 
Since the operator $A$ is the infinitesimal generator of a strongly continuous semigroup on $Y, Z$ is a Fréchet space and is also a dense subspace of $Y$; see [10, Theorem 2.3] and [5, p.53]. Moreover, it is clear from the topology on $Z$ that $\mathcal{A}$, the restriction of $A$ to $Z$, is a continuous linear mapping from $Z$ into $Z$.

For Theorem 4.2 to be applicable in the dual space $Z^{\prime}$, we require $Z$ to be a countably normed space. To this end, we define an equivalent topology on $Z$ via the countable collection of norms

$$
|\phi|_{k}:=\sum_{j=0}^{k} \alpha_{j}(\phi), \phi \in Z, k=0,1,2, \ldots
$$

To show that $Z$, equipped with this equivalent topology, is a countably normed space we must first establish the following result which is obtained by making some routine modifications to the Sobolev tower theory of Nagel $[5, \mathrm{p} .124]$.

Lemma 5.1 For each $n=1,2,3, \ldots$ and $t \geq 0$, let $Z_{n}=\left(D\left(A^{n}\right),|\cdot|_{n}\right)$ and $S_{n}(t)=\exp (t A)_{\left.\right|_{n}}$. Then

(a) each $Z_{n}$ is a Banach space;

(b) $\left\{S_{n}(t)\right\}_{t \geq 0}$ is a strongly continuous semigroup on $Z_{n}$;

(c) the infinitesimal generator, $A_{n}$, of $\left\{S_{n}(t)\right\}_{t \geq 0}$ is given by the part of $A$ in $Z_{n}$, that is

$$
\begin{aligned}
& A_{n} \phi:=A \phi, \phi \in D\left(A_{n}\right), \\
& D\left(A_{n}\right):=\left\{\phi \in Z_{n}: A \phi \in Z_{n}\right\}=D\left(A^{n+1}\right)=Z_{n+1} .
\end{aligned}
$$

Proof We first prove that (a)-(c) hold for $n=1$. Part (a) follows from the fact that $A$ is a closed operator in $Y$ and $|\cdot|_{1}$ is the associated graph norm. Also

$$
\begin{aligned}
\left|S_{1}(t) \phi\right|_{1} & =\|S(t) \phi\|_{Y}+\|A S(t) \phi\|_{Y} \\
& =\|S(t) \phi\|_{Y}+\|S(t) A \phi\|_{Y} \\
& \leq\|S(t)\|_{B(Y)}\left(\|\phi\|_{Y}+\|A \phi\|_{Y}\right)=\|S(t)\|_{B(Y)}|\phi|_{1},
\end{aligned}
$$

and

$$
\left|S_{1}(t) \phi-\phi\right|_{1}=\|S(t) \phi-\phi\|_{Y}+\|S(t) A \phi-A \phi\|_{Y} \rightarrow 0 \text { as } t \downarrow 0 .
$$

Hence $\left\{S_{1}(t)\right\}_{t \geq 0}$ is a strongly continuous semigroup on $Z_{1}$, and (c) follows from standard results on subspace semigroups [5, p.60].

Suppose now that the result is true for some fixed $n \in \mathbb{N}$. Then $\left\{S_{n}(t)\right\}_{t \geq 0}$ is a strongly continuous semigroup on $Z_{n}$ and its infinitesimal generator $A_{n}$ satisfies

$$
A_{n} \phi=A \phi, \forall \phi \in D\left(A_{n}\right)=Z_{n+1} .
$$

Let

$$
|\phi|_{n, A_{n}}:=|\phi|_{n}+\left|A_{n} \phi\right|_{n}=|\phi|_{n}+|A \phi|_{n}=\sum_{j=0}^{n}\left(\alpha_{j}(\phi)+\alpha_{j+1}(\phi)\right), \phi \in Z_{n+1}
$$


denote the graph norm on $D\left(A_{n}\right)=Z_{n+1}$. Since

$$
|\phi|_{n+1} \leq|\phi|_{n, A_{n}} \leq 2|\phi|_{n+1},
$$

$|\cdot|_{n+1}$ and $|\cdot|_{n, A_{n}}$ are equivalent norms on $Z_{n+1}$ and therefore $Z_{n+1}$ is a Banach space. Moreover,

$$
\left|S_{n+1}(t) \phi\right|_{n+1}=\sum_{j=0}^{n+1}\left\|S(t) A^{j} \phi\right\|_{Y} \leq\|S(t)\|_{B(Y)}|\phi|_{n+1}
$$

and

$$
\left|S_{n+1}(t) \phi-\phi\right|_{n+1}=\sum_{j=0}^{n+1}\left\|S(t) A^{j} \phi-A^{j} \phi\right\|_{Y} \rightarrow 0 \text { as } t \downarrow 0 .
$$

So $\left\{S_{n+1}(t)\right\}_{t \geq 0}$ is a strongly continuous semigroup on $Z_{n+1}$ and its generator is the part of $A_{n}$ in $Z_{n+1}$; that is

$$
\begin{aligned}
& A_{n+1} \phi:=A_{n} \phi, \phi \in D\left(A_{n+1}\right), \\
& D\left(A_{n+1}\right):=\left\{\phi \in D\left(A_{n}\right) \cap Z_{n+1}: A_{n} \phi \in Z_{n+1}\right\}=\left\{\phi \in Z_{n+1}: A \phi \in Z_{n+1}\right\} .
\end{aligned}
$$

The results follow by induction.

Lemma $5.2 Z_{n}=\overline{\left(Z,|\cdot|_{n}\right)}$ for each $n=1,2,3, \ldots$

Proof Let $D^{\infty}\left(A_{n}\right)=\cap_{k \geq 0} D\left(A_{n}^{k}\right)$. From [5, Proposition 1.8], $D^{\infty}\left(A_{n}\right)$ is dense in $Z_{n}$ and the stated result follows on noting that $A_{n}$ is the restriction of $A$ to $Z_{n}$.

Thus the Fréchet space $Z$ and Banach spaces $Z_{n}$ satisfy (4.1) - (4.4), that is

$$
\begin{aligned}
& |\phi|_{0} \leq|\phi|_{1} \leq|\phi|_{2} \leq \cdots \forall \phi \in Z ; Y=Z_{0} \supset Z_{1} \supset Z_{2} \supset \cdots \supset Z_{k} \supset \cdots ; \\
& Z_{k}=\overline{\left(Z,\left.|\cdot|\right|_{k}\right)} \text { for each } k ; Z=\cap_{k \geq 0} Z_{k} .
\end{aligned}
$$

We define the dual space, $Z^{\prime}$, of $Z$, equipped with the weak*-topology, to be the associated space of generalized functions. Note that, from [7, p.34], $f \in Z^{\prime}$ if and only if there exists some non-negative integer $r$ and constant $C$ such that

$$
|\langle f, \phi\rangle| \leq C|\phi|_{r} \forall \phi \in Z .
$$

The least such $r$ is called the order of the functional $f$. Given $\psi \in X$ if we define $\tilde{\psi}$ on $Z$ by (2.26), then

$$
|\langle\tilde{\psi}, \xi\rangle| \leq\|\psi\|_{X}\|\xi\|_{Y}=\|\psi\|_{X}|\xi|_{0}, \forall \xi \in Z
$$

and so $\psi$ generates a regular generalized function of order 0 in $Z^{\prime}$. Similarly, if $q_{0} \in C([0, \infty), \mathbb{R})$, then

$$
\begin{aligned}
& \left|\left\langle q_{0}(t) \mathbf{1}(\mathbf{u}) \otimes \delta\left(\mathbf{x}-\mathbf{x}_{0}\right), \xi\right\rangle\right| \\
= & \left|q_{0}(t) \int_{S} \xi\left(\mathbf{x}_{0}, \mathbf{u}\right) d \mathbf{u}\right| \\
\leq & 4 \pi\left|q_{0}(t)\right||\xi|_{0}, \quad \forall t \geq 0, \xi \in Z,
\end{aligned}
$$


and so the localized point source is also a generalized function of order 0 in $Z^{\prime}$. Lastly, for each $\psi \in D(T)$, we require

$$
\begin{aligned}
\langle\tilde{T} \tilde{\psi}, \eta\rangle & :=\langle\widetilde{T \psi}, \eta\rangle \\
& =(\eta, T \psi)=(\mathcal{A} \eta, \psi)=\langle\tilde{\psi}, \mathcal{A} \eta\rangle, \forall \eta \in Z
\end{aligned}
$$

where we have used (3.17) and this leads to the definition

$$
\langle\tilde{T} f, \eta\rangle:=\langle f, \mathcal{A} \eta\rangle \forall f \in Z^{\prime}, \eta \in Z
$$

Thus $\tilde{T}=\mathcal{A}^{\prime}$, where $\mathcal{A}^{\prime}$ denotes the adjoint of $\mathcal{A}$ on the complete countably normed space $Z$, and therefore, from standard results on adjoints, $\tilde{T}$ is well-defined as a continuous linear mapping from $Z^{\prime}$ into $Z^{\prime}$.

\section{Solution of the time-dependent point source problem}

The results of the previous sections can now be applied to establish the existence of a unique solution $N:[0, \infty) \rightarrow Z^{\prime}$ of the abstract Cauchy problem

$$
\frac{d}{d t} N(t)=\tilde{T}[N(t)]+q_{0}(t) \mathbf{1}(\mathbf{u}) \otimes \delta\left(\mathbf{x}-\mathbf{x}_{0}\right) ; u(0)=u_{0} \in Z^{\prime}
$$

where $\tilde{T}=\mathcal{A}^{\prime}$.

Lemma 6.1 The operator $\mathcal{A}$ is the infinitesimal generator of a uniquely defined equicontinuous semigroup, $\{\exp (t \mathcal{A})\}_{t \geq 0}$ of class $C_{0}$ in $Z$. Moreover,

$$
e^{t \mathcal{A}} \phi=e^{t A} \phi, \forall \phi \in Z
$$

where $\{\exp (t A)\}_{t \geq 0}$ is the semigroup on $Y$ generated by $A$ (see Section 3).

Proof For each $k=0,1,2, \ldots$ and $\phi \in Z$,

$$
\begin{aligned}
& \alpha_{k}\left(\left[I-n^{-1} \mathcal{A}\right]^{-1} \phi\right) \\
= & \alpha_{k}\left(n[n I-\mathcal{A}]^{-1} \phi\right) \\
\leq & \frac{n}{n+c\left(\sigma_{\min }-\sigma_{s, \text { max }}\right)} \alpha_{k}(\phi) \leq \alpha_{k}(\phi),
\end{aligned}
$$

since $\sigma_{\min }-\sigma_{s, \max }$ is assumed to be positive. Consequently

$$
\alpha_{k}\left(\left[I-n^{-1} \mathcal{A}\right]^{-m} \phi\right) \leq \alpha_{k}(\phi), \forall n=1,2,3, \ldots, m=0,1,2, \ldots
$$

and so, from [18, p. 246], $\mathcal{A}$ is the infinitesimal generator of an equicontinuous semigroup of class $C_{0}$ on $Z$. Moreover, from [18, p. 248],

$$
e^{t \mathcal{A}} \phi=\lim _{n \rightarrow \infty} \exp \left(t \mathcal{A}\left[I-n^{-1} \mathcal{A}\right]^{-1}\right) \phi, \forall \phi \in Z
$$


Since $\mathcal{A}$ is the restriction of $A$ to $Z$ and convergence in $Z$ implies convergence in $Y$, we obtain

$$
e^{t \mathcal{A}} \phi=\lim _{n \rightarrow \infty} \exp \left(t \mathcal{A}\left[I-n^{-1} A\right]^{-1}\right) \phi=e^{t A} \phi
$$

Theorem 6.2 Let $q_{0} \in C^{1}([0, \infty), \mathbb{R})$. Then equation (6.1) has a unique solution $N:[0, \infty) \rightarrow Z^{\prime}$ for any $u_{0} \in Z^{\prime}$.

Proof A routine calculation shows that the function $q:[0, \infty) \rightarrow Z^{\prime}$ defined by $q(t)=q_{0}(t) \mathbf{1}(\mathbf{u}) \otimes \delta\left(\mathbf{x}-\mathbf{x}_{0}\right)$ is (weak*-) differentiable with (weak*-) continuous derivative $\frac{d}{d t}\left[q_{0}(t)\right] \mathbf{1}(\mathbf{u}) \otimes \delta\left(\mathbf{x}-\mathbf{x}_{0}\right)$. It now follows from Theorem 4.2 that the unique solution is given by

$$
\begin{aligned}
u(t) & =e^{t \mathcal{A}^{\prime}} u_{0}+\int_{0}^{t} e^{(t-s) \mathcal{A}^{\prime}} q(s) d s \\
& =e^{t \tilde{T}} u_{0}+\int_{0}^{t} e^{(t-s) \tilde{T}} q_{0}(s) \mathbf{1}(\mathbf{u}) \otimes \delta\left(\mathbf{x}-\mathbf{x}_{0}\right) d s
\end{aligned}
$$

\section{Conclusion}

The distributional approach presented in this paper has led to mathematically rigorous existence and uniqueness results for photon transport problems with time-dependent point sources. Although the method involves rather technical material, such as countably normed spaces and equicontinuous semigroups on locally convex spaces, we believe that it has certain advantages over a Banach-space based approach, and has the potential to be exploited further. Firstly, the constructive manner in which the "test function" space $Z$ is obtained from the infinitesimal generator $T$ of a contractive semigroup on an $L^{1}$ space lends itself to other similar evolution equations with sources or initial conditions expressed in terms of singular distributions. A particular case which we intend to explore in a future paper is that of a fragmentation equation with power-law kernels and mono-disperse initial conditions; see [12]. Secondly, it is worth emphasizing that the method guarantees that the infinitesimal generator $\mathcal{A}$ of the equicontinuous semigroup on $Z$ is continuous and defined on all of $Z$. This should be compared with the usual Banach-space situation where closed and densely-defined generators are customary. Thirdly, the additional structure provided by the use of countably normed spaces means that $Z$ and $Z^{\prime}$ can be expressed in the form

$$
Z=\bigcap_{n=0}^{\infty} Z_{n}, \quad Z^{\prime}=\bigcup_{n=0}^{\infty} Z_{n}^{*},
$$

where $\left(Z_{n},|\cdot|_{n}\right)=\overline{\left(Z,|\cdot|_{n}\right)}$, with $|\cdot|_{n}$ defined via (5.1), and $Z_{n}^{*}$ is the Banach space of all continuous linear functionals on $Z_{n}$; see [7, pp. 17, 36]. Since $Z_{0}=Y$, the Banach space of measures that features in the work of Suhadolc and Vidav emerges in our approach as the space $Z_{0}^{*}$. Finally, it is well-known that the Dirac 
delta distribution can be obtained as a limit of a sequence of suitably defined classical functions such as the "cap-shaped" functions

$$
\omega_{n}(\mathbf{x})= \begin{cases}C_{n} \exp \left(1 /\left(1-n^{2}|\mathbf{x}|^{2}\right)\right) & \text { when }|\mathbf{x}| \leq 1 / n \\ 0 & \text { when }|\mathbf{x}|>1 / n\end{cases}
$$

where $C_{n}$ is a normalizing constant; see [16, p.74]. For each sufficiently large $n, \omega_{n}$ generates a regular generalized function $\mathbf{1}(\mathbf{u}) \otimes \tilde{\omega}_{n}\left(\mathbf{x}-\mathbf{x}_{0}\right)$, of order 0 , in $Z^{\prime}$ and it is not difficult to show that $\mathbf{1}(\mathbf{u}) \otimes \tilde{\omega}_{n}\left(\mathbf{x}-\mathbf{x}_{0}\right) \rightarrow \mathbf{1}(\mathbf{u}) \otimes \delta\left(\mathbf{x}-\mathbf{x}_{0}\right)$ in $Z^{\prime}$. This suggests the possibility that the solution $N$ of equation (6.1) for certain initial conditions $u_{0}$ may be obtained as an appropriate limit of solutions $N_{n}$ of the "classical" problem

$$
\frac{d}{d t} N_{n}(t)=T\left[N_{n}(t)\right]+q_{0}(t) \mathbf{1}(\mathbf{u}) \otimes \omega_{n}\left(\mathbf{x}-\mathbf{x}_{0}\right) ; u(0)=u_{0}
$$

This also is a topic which we hope to investigate further.

\section{References}

[1] A. Belleni-Morante and A.C. McBride, Applied Nonlinear Semigroups (Wiley, Chichester, 1998).

[2] A. Belleni-Morante, W. Lamb and A.C. McBride, Photon transport problems involving a point source, Analysis and Applications, 5 (2007) 77-93.

[3] M.A. Dopita and R.S. Sutherland, Astrophysics of the Diffuse Universe (Springer-Verlag, Berlin, 2003).

[4] J. Dyson and D.A. Williams, The Physics of the Interstellar Medium (Inst. of Physics Pub., Bristol, 1997).

[5] K-J Engel and R. Nagel, One Parameter Semigroups for Linear Evolution Equations (Springer, New York, $2000)$.

[6] B. Farkas, Perturbations for bi-continuous semigroups, Studia Math., 161 (2004) 147-161.

[7] I.M. Gel'fand and G.E. Shilov, Generalized Functions (Vol. 2) (Academic Press, New York and London, 1968).

[8] W. Greenberg, C. van der Mee and V. Protopopescu, Boundary Value Problems in Abstract Kinetic Theory (Birkhäuser, Basel, 1987).

[9] F. Kühnemund, A Hille-Yosida theorem for bi-continuous semigroups, Semigroup Forum, 67 (2003) 205225 .

[10] W. Lamb and D.F. McGhee, Spectral theory and functional calculus on spaces of generalized functions, J. Math. Anal. Appl., 163 (1992) 238-260.

[11] T. Lant and H. Thieme, Markov transition functions and semigroups of measures, Semigroup Forum, 74 (2007) 337-369. 
[12] E.D. McGrady and R.M. Ziff, "Shattering" transition in fragmentation, Phys. Rev. Lett., 58 (1987) 892895.

[13] D. Mihalas and B. Weibel Mihalas, Foundations of Radiation Hydrodynamics (Dover, New York, 2000).

[14] G.C. Pomraning, The Equations of Radiation Hydrodynamics (Pergamon Press, Oxford, 1973).

[15] A. Suhadolc and I. Vidav, Linearized Boltzmann equation in spaces of measures, Mathematica Balkanica, 3 (1973) 514-529.

[16] V.S. Vladimirov, Equations of Mathematical Physics (Marcel Dekker, New York, 1971).

[17] J. Voigt, Functional Analytic Treatment of the Initial Boundary Value Problem for Collisionless Gases, Habilitationsschrift, Ludwig Maximilians Universität, München (1980).

[18] K. Yosida, Functional Analysis 6th edn. (Springer-Verlag, Berlin, 1980).

[19] A.H. Zemanian, Generalized Integral Transformations (Interscience, New York, 1968). 